\title{
Review of: "Microbial associations with Microscopic Colitis"
}

Ricardo Araujo

Potential competing interests: The author(s) declared that no potential competing interests exist.

This manuscript confirms the results of previous publications regarding microscopic colitis and adds a few new details on the topic. In this study the authors reported the importance of the genus Collinsella; the family Coriobacteriaceae was described as relevant in previous studies so this is a small addition to what was previously known. Samples from ascending and descending colon were described as presenting microbiomes and this is a valuable information. The authors considered in this manuscript a high number of control individuals.

Some suggestion to improve the manuscript:

1) Details regarding diet and medications received by the patients were not provided. Please consider such information for the results analyses and discussion.

2) The sequencing analysis considered only V2 region of $16 \mathrm{~S}$ rRNA gene. Most of the previous studies used V1-V3 or V3-V4 regions. Please add references supporting the use of V2 region for microbiome evaluation.

3) Table 2 can se moved to supplements.

4) Figure 1: graphs b, c g and h can be removed.

5) Figures 2 and 3 show exactly the same results, so Figure 3 can be removed. 